\title{
Imaging Taurine in the Central Nervous System using Chemically Specific Imaging at the Sulfur K-edge
}

\author{
Mark J. Hackett, ${ }^{\dagger, \|}$ Phyllis G. Paterson, ${ }^{\dagger}$ Ingrid J. Pickering, ${ }^{*, \dagger, \S}$ and Graham N. George ${ }^{*, \dagger, \S}$ \\ ${ }^{\dagger}$ Molecular and Environmental Sciences Group, Department of Geological Sciences, University of Saskatchewan, \\ 114 Science Place, Saskatoon, Saskatchewan S7N 5E2, Canada. E-mail: g.george@usask.ca; \\ ingrid.pickering@usask.ca; Tel: +1 306966 5722; +1 3069665706 \\ College of Pharmacy and Nutrition, University of Saskatchewan, 107 Wiggins Rd, Saskatoon, Saskatchewan, S7N \\ 5E5, Canada \\ ${ }^{\S}$ Department of Chemistry, University of Saskatchewan, 110 Science Place, Saskatoon, Saskatchewan S7N 5C9, \\ Canada \\ "Present address: Curtin University, Perth WA 6845, Australia.
}

\section{Supporting Information}

\author{
Table S1-S3
}

Figures S1-S3

*Corresponding Authors: ingrid.pickering@usask.ca, graham.george@usask.ca 
Table S1. Normalized intensities of model compounds used for chemically specific sulfur imaging

\begin{tabular}{ccccccc}
\hline $\begin{array}{c}\text { Energy } \\
(\mathbf{e V})\end{array}$ & RSSR & RSH & RSR & Sulfoxide & $\begin{array}{c}\text { Sulfonic } \\
\text { Acid }\end{array}$ & $\begin{array}{c}\text { Sulfate } \\
\text { Ester O- }\end{array}$ \\
\hline 2469.85 & 2.78 & 1.47 & 0.72 & 0.08 & 0.05 & 0.01 \\
2470.55 & 1.82 & 3.04 & 2.48 & 0.11 & 0.05 & 0.01 \\
2470.8 & 1.84 & 2.71 & 3.20 & 0.13 & 0.05 & 0.02 \\
2473.5 & 0.77 & 1.12 & 1.01 & 4.52 & 0.09 & 0.04 \\
2474.4 & 0.83 & 1.17 & 1.08 & 2.44 & 0.11 & 0.06 \\
2478.2 & 1.23 & 1.23 & 1.34 & 0.98 & 5.40 & 3.20 \\
2479.2 & 1.17 & 1.16 & 1.28 & 1.06 & 2.75 & 4.94 \\
2479.6 & 1.14 & 1.12 & 1.23 & 1.07 & 1.92 & 5.79 \\
2479.8 & 1.12 & 1.11 & 1.21 & 1.07 & 1.62 & 6.10 \\
\hline
\end{tabular}

Table S2. Micro-XAS analysis of the speciation of sulfur within different tissue layers of the cerebellum ${ }^{a}$

\begin{tabular}{lccc}
\hline Tissue & IL & GL & ML \\
\hline Thiol & $32(5) \pm 3$ & $37(4) \pm 2$ & $31(6) \pm 5$ \\
Thio-Ether & $26(4) \pm 3$ & $43(4) \pm 1^{*}$ & $45(4) \pm 1^{*}$ \\
Disulfide & $2.9(2) \pm 0.5$ & $0.5(2) \pm 0.77$ & $4.4(3) \pm 2.6$ \\
Sulfoxide & $2.1(8) \pm 0.2$ & $3.1(7) \pm 1.5$ & $3.8(9) \pm 1.8$ \\
Sulfonic Acid & $11.6(1) \pm 1.8$ & $15.1(3) \pm 1.2^{*}$ & $15.2(1) \pm 1.2^{*}$ \\
Sulfate Ester & $25.1(1) \pm 1.9$ & $0.9(8) \pm 0.5^{* *}$ & $1.2(9) \pm 1.6^{* *}$ \\
\hline
\end{tabular}

${ }^{a} \mathrm{IL}=$ Inner Layer (white matter), GL = Granular Layer (grey matter), ML = Molecular Layer. Representative spectra and fits are shown in Fig. 2. Values given are the average percentages \pm standard deviation of replicate measurements $(n=4)$. The values in parenthesis are the average estimated standard deviation from the individual fits (given as the last digit of the percentage) obtained from the diagonal elements of the covariance matrices. The estimated standard deviation gives an indication of how well each component is determined whereas the standard of the triplicate measurements gives an indication of how much variation is present between different samples. We note that as expected for slightly different individual fits, the average percentages do not total to $100 \%$, although the individual fits do total to $100 \%$. A significant difference in average \% composition values of grey matter and molecular layer tissue relative to white matter tissue was determined with the Student's t-test ${ }^{*} p<0.05,{ }^{* *} p<0.01$ ). Using the same approach, no significant differences in the speciation of sulfur were found between grey matter and molecular layer tissue. 
Table S3. Micro-XAS analysis of the speciation of sulfur within vulnerable (CA1) and less vulnerable (CA3) subsectors of the rat hippocampus following global brain ischemia ${ }^{a}$

\begin{tabular}{lccc}
\hline Tissue & Ischemic CA1 & Ischemic CA3 & Sham CA1 \\
\hline Thiol & $18(4) \pm 11^{*, * *}$ & $32(4) \pm 11$ & $40(2) \pm 10$ \\
Thio-Ether & $60(3) \pm 12^{*, \dagger \dagger}$ & $38(3) \pm 9$ & $32(2) \pm 11$ \\
Disulfide & $4.6(2) \pm 1.0^{*, * *}$ & $2.0(8) \pm 1.3$ & $2.7(8) \pm 1.0$ \\
Sulfoxide & $4.4(6) \pm 0.8$ & $5.0(5) \pm 1.6$ & $3.9(4) \pm 1.2$ \\
Sulfonic Acid & $9.3(7) \pm 1.4^{\dagger, * *}$ & $17.1(1) \pm 2.9$ & $19.0(1) \pm 1.4$ \\
Sulfate Ester & $3.6(6) \pm 0.7$ & $6.0(4) \pm 2.7$ & $2.9(4) \pm 2.0$ \\
\hline
\end{tabular}

${ }^{a}$ Values given are the average percentages \pm standard deviation of replicate measurements $(n=5)$. The values in parenthesis are the average estimated standard deviation from the individual fits (given as the last digit of the percentage) obtained from the diagonal elements of the covariance matrices. The estimated standard deviation gives an indication of how well each component is determined whereas the standard of the triplicate measurements gives an indication of how much variation is present between different samples. We note that as expected for slightly different individual fits, the average percentages do not total to $100 \%$, although the individual fits do total to $100 \%$. A significant difference in average \% composition values of the CA1 subsector of sham animals relative to ischemic animals was determined with the Student's t-test $\left({ }^{*} p<0.05,{ }^{\dagger} p<0.01\right)$. A significant difference in average $\%$ composition values of the CA3 subsector of ischemic animals relative to the CA1 subsector of ischemic animals was determined with a paired t-test $\left(* * p<0.05,{ }^{*} \mathrm{p}<0.01\right)$. 


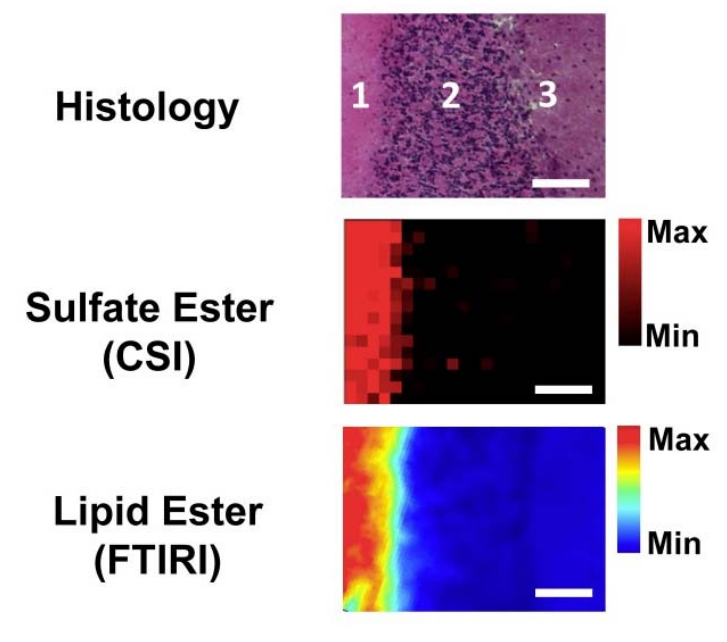

Figure S1. Chemically specific sulfur imaging of sulfatides and Fourier transform infared imaging (FTIRI) of lipids, which co-localize in white matter. Inner layer white matter (1), granular layer grey matter (2), molecular layer (3). Scale bar $=100 \mu \mathrm{m}$. A subset of samples prepared on $\mathrm{Si}_{3} \mathrm{~N}_{4}$ membranes were analyzed with FTIRI prior to chemically specific XFI analysis at the APS, to demonstrate the complementary capabilities of FTIRI and chemically specific sulfur imaging. FTIRI was performed at the Mid-IR beamline at the CLS in "offline" mode with a Hyperion 3000 microscope fitted with an upper objective of $15 \times$ magnification and a numerical aperture of 0.4 , combined with a lower condenser of $15 \times$ magnification and 0.4 numerical aperture. This arrangement yielded a pixel size of $2.67 \mu \mathrm{m}$. A focal plane array detector was illuminated with a thermal globar source. FTIRSpectroscopic images were collected across the spectral region $3600-900 \mathrm{~cm}^{-1}$, with a spectral resolution of $4 \mathrm{~cm}^{-1}$ with the co-addition of 128 scans, with a background image similarly collected from blank substrate using 128 coadded scans. The background was collected immediately prior to each sample. Images of the total lipid ester distribution were calculated from the integrated area under the ester carbonyl band $v(\mathrm{C}=\mathrm{O})$ from $1755-1715 \mathrm{~cm}^{-1}$. All data processing and image generation were performed using Cytospec software (Cytospec, Version 1.2.04) and Opus software (Version 6.5, Bruker, Ettlingen, Germany). 


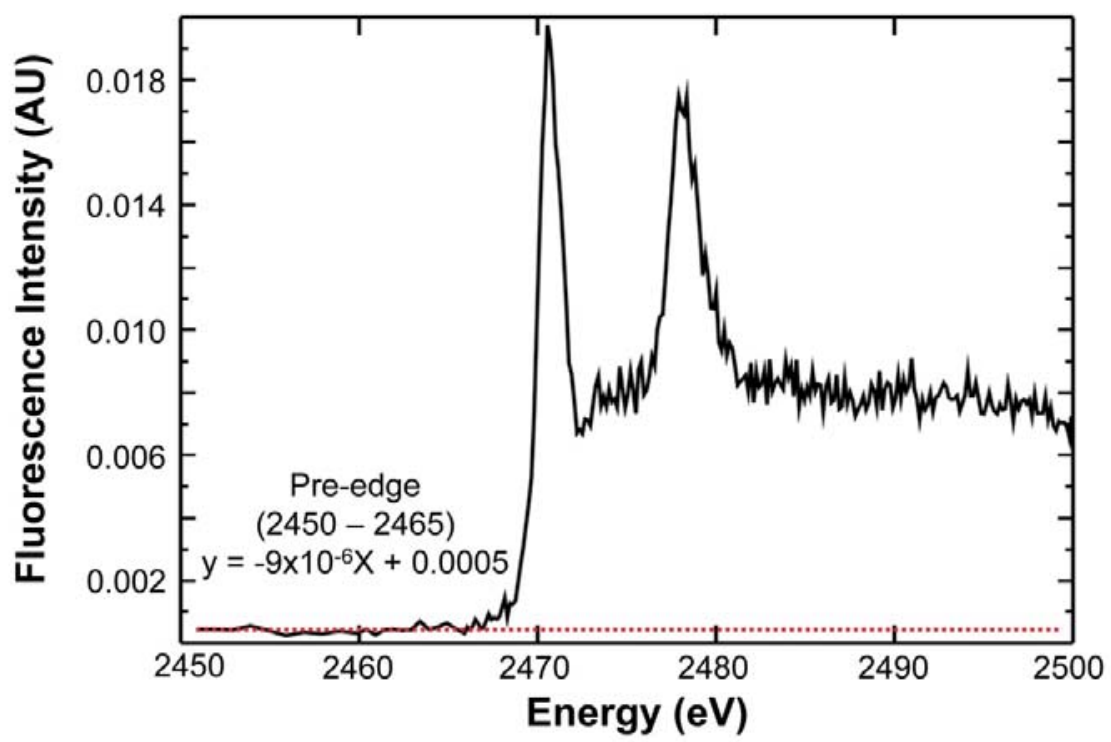

Figure S2. The pre-edge region of micro-XAS spectra at the sulfur K-edge is linear with negligible slope (red dashed line). A representative example is shown, where a linear fit can be applied to the pre-edge region (2450-2465 $\mathrm{eV}$ ), which results in a trend line with a slope of $-9 \times 10^{-6}$.

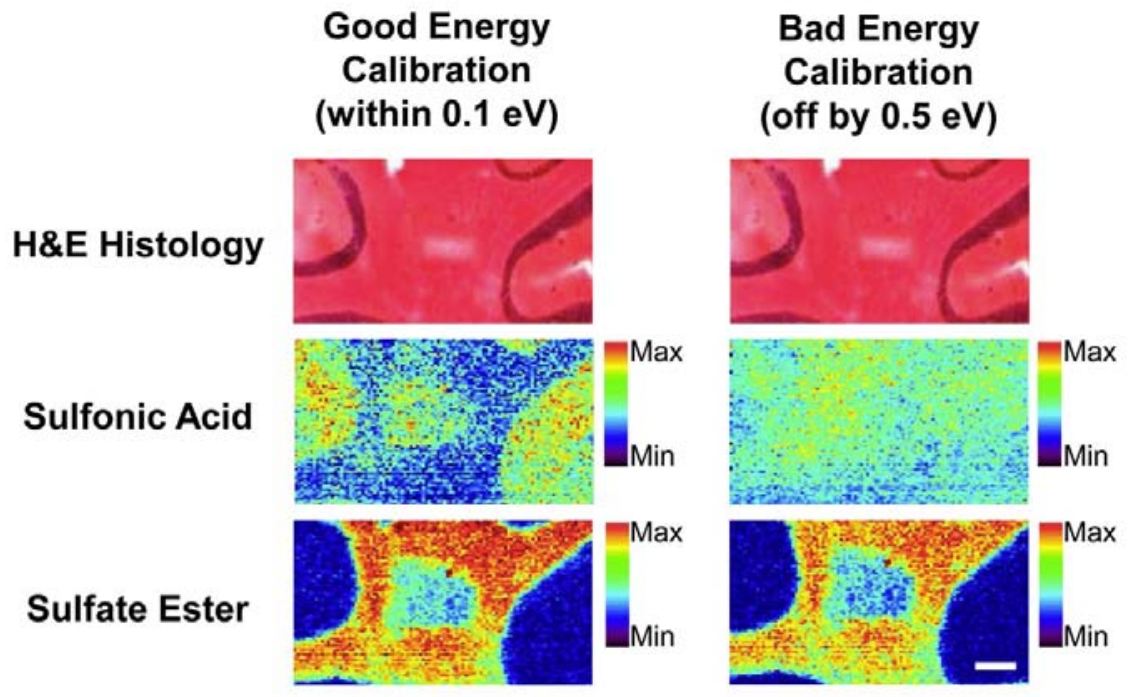

Figure S3. Effect of energy calibration / stability on chemically specific images of the cerebellum. An error in calibration of just $0.5 \mathrm{eV}$ leads to erroneous conclusions about the distribution of sulfonic acid, which appears almost uniform in the right hand panel but much more distinct in the left. The sulfate ester map from the miscalibrated dataset is also more noisy than its counterpart, although the distribution is more apparent. 